\title{
Surface corona-bar discharges for production of pre-ionizing UV light for pulsed high-pressure plasmas
}

\author{
Zhongmin Xiong and Mark J Kushner ${ }^{1}$ \\ University of Michigan, Electrical Engineering and Computer Science Department, 1301 Beal Ave, \\ Ann Arbor, MI 48109-2122, USA \\ E-mail: zxiong@umich.edu and mjkush@umich.edu
}

Received 17 September 2010, in final form 1 November 2010

Published 2 December 2010

Online at stacks.iop.org/JPhysD/43/505204

\begin{abstract}
Multi-atmospheric pressure, pulsed electric discharge excited lasers require pre-ionization to produce spatially uniform glows. Many such systems use corona bars to produce ultraviolet (UV) and vacuum ultraviolet (VUV) light as photo-ionization sources for this purpose. Corona bars are transient surface discharges, typically in a cylindrical geometry, that sustain high electron temperatures and so are efficient UV and VUV sources. In this paper, results from a numerical study of surface corona-bar discharges in a multi-atmosphere pressure $\mathrm{Ne} / \mathrm{Xe}$ gas mixture are discussed. The discharge consists of a high-voltage electrode placed on the surface of a corona bar which is a dielectric tube surrounding a cylindrical metal electrode. After the initial breakdown an ionization front propagates along the circumference of the corona bar and produces a thin plasma sheet near the dielectric surface. The propagation speed of the ionization front ranges from $2 \times 10^{7}$ to $3.5 \times 10^{8} \mathrm{~cm} \mathrm{~s}^{-1}$, depending on the applied voltage and dielectric constant of the corona-bar insulator. As the discharge propagates around the circumference, the surface of the corona-bar is charged. The combined effects of surface curvature and charge deposition result in a non-monotonic variation of the electric field and electron temperature as the ionization front traverses the circumference. The UV fluxes collected on a surrounding circular surface correlate with the motion of the ionization front but with a time delay due to the relatively long lifetime of the precursor to the emitting species $\mathrm{Ne}_{2}^{*}$.
\end{abstract}

\section{Introduction}

In transversely excited, multi-atmospheric pressure gas discharge lasers, such as excimer lasers, maintaining the discharge in a volumetrically diffuse or glow mode is critical to efficient laser pumping and having high optical quality [1]. Obtaining a glow discharge mode typically requires preionization of the discharge to electron densities of $10^{9}-$ $10^{10} \mathrm{~cm}^{-3}$ [2]. This is typically accomplished by using an ultraviolet (UV), vacuum ultraviolet (VUV) or x-ray radiation source. Sufficient pre-ionization prevents the formation of streamers and their subsequent transition to arcs [3]. The density and uniformity of the photo-ionization-produced electrons as well as the timing of the pre-ionization with respect

\footnotetext{
${ }^{1}$ Author to whom any correspondence should be addressed.
}

to application of high voltage to the discharge electrodes directly affect the laser's performance [4].

UV or VUV radiation for pre-ionization can be produced by arrays of spark discharges or by corona discharges sustained over a dielectric surface, often called a corona bar. (We will collectively refer to both UV and VUV photon fluxes as UV fluxes.) A corona-bar pre-ionization source typically consists of an annular dielectric tube with a grounded metal cylinder along its centre. The axis of the corona bar is placed parallel to the axis of the laser discharge in order to illuminate the gap between the electrodes with UV photons. A high voltage electrode, typically with a point contact on the dielectric surface in the axial direction, triggers and sustains the corona discharge. The corona discharge begins at the trigger electrode and propagates along the circumference of 
the dielectric surface, analogous to a corona streamer in the gas phase. Excited states produced by the avalanche front are the precursors for producing the pre-ionizing UV light [5]. In addition to producing UV light for pre-ionization, surface corona discharges are also widely used in lighting [6], fluorescence spectroscopy [7], waste treatment [8], chemical synthesis, surface modification, functionalization [9] and more recently for active aerodynamic flow control $[10,11]$.

Surface corona discharges are a combination of a corona discharge and a dielectric barrier discharge (DBD). The propagation of the discharge along the surface is similar to that of streamers in bulk gases, but is modified by the presence of the surface through polarization of the surface material, intensification of the electric field and release of secondary electrons. The similarity to gas phase streamers is in the space charge enhanced electric field at the head of the avalanche front that propagates in the plasma channel. The similarity to DBDs is in the adjacency of a dielectric surface which is charged by the plasma channel. Experimentally, the interaction between such a discharge and surface has been characterized using the speed of propagation of the plasma channel and its dependence on voltage, pressure and the dielectric constant of the surface material. For example, Hidaka and Murooka [12] investigated surface discharge development in a needle-to-disc configuration at atmospheric pressure in air and found the discharge propagation speed to be up to $8.4 \times 10^{8} \mathrm{~cm} \mathrm{~s}^{-1}$ for negative streamers and $1.5 \times 10^{9} \mathrm{~cm} \mathrm{~s}^{-1}$ for positive streamers. Hong [13] studied corona discharges over a cylindrical surface in multi-atmospheric pressure $\mathrm{Ne}$ and found the azimuthal discharge propagation speed to be in the range $10^{7}-10^{8} \mathrm{~cm} \mathrm{~s}^{-1}$. Walter [14] investigated surface corona discharges having different dielectric materials and showed that higher dielectric constants lead to better laser performance by allowing more energy deposition into the gas mixture. Recent experiments by Kozlov et al [15] found that not only the dielectric constant but surface properties, thickness and structure of the dielectric layer play significant roles in the appearance and development of the discharge.

Rutkevich [16] analysed the structure of sliding discharges over a flat dielectric surface based on a self-similar formulation. They found a quantitative agreement between the calculated and experimental propagation speed as a function of the applied voltage, although the propagation speed in the experiments is not a constant as assumed in the model but decreases with propagation distance. Sobota et al [17] modelled pulse discharges in Ar near a flat dielectric surface and concluded that the bulk gas processes alone cannot account for the discharge propagation in the vicinity of the surface. In the context of aerodynamic flow control, Boeuf and Pitchford [18] numerically investigated micro-discharges in an asymmetric surface DBD. They found that the propagation speed of the sliding ionization wave is on the order of $3 \times 10^{6} \mathrm{~cm} \mathrm{~s}^{-1}$ when including only the secondary emission by electron impact as the electron generation mechanism ahead of the front.

In this paper, we present results from a numerical investigation of a corona discharge propagating over a cylindrical dielectric surface in a multi-atmosphere $\mathrm{Ne} / \mathrm{Xe}$ mixture. The context is the use of corona bars for the production of pre-ionizing UV light, as might be used in a pulsed discharge laser. The electrical properties of the discharges are discussed and correlated with the production of visible and UV radiation. We found that the propagation speed of the ionization front ranges from $2 \times 10^{7}$ to $3.5 \times 10^{8} \mathrm{~cm} \mathrm{~s}^{-1}$, depending on the applied voltage and dielectric constant of the corona bar. The variations of the electric field and electron temperature in the ionization front are non-monotonic as the front traverses the circumference of the corona bar. The governing equations and the model are briefly described in section 2 along with the reaction mechanism used for $\mathrm{Ne} / \mathrm{Xe}$ mixtures. In sections 3-5, a discussion of the discharge dynamics and the UV production are presented. Section 6 contains our concluding remarks.

\section{Description of the model}

The modelling of the cylindrical surface corona discharge is based on nonPDPSIM which is a two-dimensional plasma hydrodynamics model with radiation transport. Continuity equations for charged and neutral species, and Poisson's equation are solved coincidently with the electron energy equation with transport coefficients obtained from solutions of Boltzmann's equation. A Monte Carlo simulation is used to track sheath accelerated electrons produced by secondary processes at the surface. The spatial discretization is based on finite volume methods on an unstructured mesh and the time integration is implemented using a fully implicit Newton iteration method. The details of the model are described elsewhere [19], but briefly the following equations are solved for charge species,

$$
\begin{gathered}
-\nabla \cdot \varepsilon \nabla \Phi=\sum_{j} n_{j} q_{j}+\rho_{\mathrm{s}}, \\
\frac{\partial N_{i}}{\partial t}=-\nabla \cdot \vec{\phi}_{i}+S_{i}, \\
\frac{\partial \rho_{\mathrm{s}}}{\partial t}=\sum_{i}-\nabla \cdot\left(q_{i} \vec{\phi}_{i}\left(1+\gamma_{i}\right)\right)+\nabla \cdot(\sigma \nabla \Phi),
\end{gathered}
$$

where $\varepsilon, \Phi, \rho_{\mathrm{s}}, N, \phi, \gamma, \sigma, S$ and $q$ refer to the permittivity, electric potential, surface charge density, charged species number density, species flux, secondary electron emission coefficient, conductivity of the solid materials, source terms and elementary charge, respectively. The source term $S_{i}$ contains the production and loss of species $i$ due to electron impact ionization and excitation, heavy particle reactions, photo-ionization, secondary emission and surface reactions. The charged particle fluxes $\phi_{i}$ are approximated with the Scharfetter-Gummel technique [20] and equations (1)-(3) are simultaneously solved in each Newton iteration step. Once the charged particle densities are updated, the electron energy equation is integrated for average energy $\varepsilon$

$$
\frac{\partial}{\partial t}\left(n_{\mathrm{e}} \varepsilon\right)=q \vec{\phi}_{\mathrm{e}} \cdot \vec{E}-n_{\mathrm{e}} \sum_{i} N_{i} k_{i} \Delta \varepsilon_{i}-\nabla \cdot\left(\frac{5}{2} \varepsilon \vec{\phi}_{\mathrm{e}}-\lambda \nabla T_{\mathrm{e}}\right),
$$

where $n_{\mathrm{e}}$ is electron density, $E=-\nabla \Phi$ is the electric field, $k_{i}$ is the rate coefficient for collision process $i$ with species 
having density $N_{i}$ and energy loss $\Delta \varepsilon_{i}, \lambda$ is the electron thermal conductivity and $\vec{\phi}_{\mathrm{e}}$ is the electron flux and $T_{\mathrm{e}}$ is the electron temperature defined as $(2 \varepsilon / 3)$.

During the short duration of the corona discharge, advective motion of neutral species by temperature or pressure gradients is not important. In the majority of our simulations, the densities of neutral species are given by their continuity equations with only diffusion for transport. These continuity equations for neutral species are solved in a time-slicing manner with the charged particle continuity equations,

$$
\frac{\partial N_{i}}{\partial t}=-\nabla \cdot\left(-D_{i} N_{\mathrm{T}} \nabla\left(\frac{N_{i}}{N_{\mathrm{T}}}\right)\right)+S_{i},
$$

where the $N_{i}$ is the density of neutral species $i, N_{\mathrm{T}}$ is the total gas density, $D_{i}$ is the diffusion coefficient and $S_{i}$ is the source or sink due to volumetric or surface processes. For comparison purposes, the fully compressible Navier-Stokes equations are solved for the fluid averaged density, momentum and gas temperature. The implementation of these equations in nonPDPSIM is described in [21].

Photon transport in the plasma is accounted for using a propagator method. The photo ionization source for species $m$ at location $\vec{r}_{i}$ due to the emission of photons at location $\vec{r}_{j}$ by species $k$ is

$$
\begin{gathered}
S_{m}\left(\vec{r}_{i}\right)=\sigma_{k m}^{\mathrm{I}} N_{m}\left(\vec{r}_{i}\right) A_{k} \int N_{k}\left(\vec{r}_{j}^{\prime}\right) G_{k}\left(\vec{r}_{j}^{\prime}, \vec{r}_{i}\right) \mathrm{d}^{3} \vec{r}_{j}^{\prime}, \\
G_{k}\left(\vec{r}_{j}^{\prime}, \vec{r}_{i}\right)=\frac{\exp \left(-\int_{\vec{r}_{j}^{\prime}}^{\vec{r}_{i n}^{\prime}} \sum_{l} \sigma_{l k} N_{l}\left(\vec{r}_{n}^{\prime}\right) \mathrm{d} \vec{r}_{n}^{\prime}\right)}{4 \pi\left|\vec{r}_{i}^{\prime}-\vec{r}_{j}\right|^{2}},
\end{gathered}
$$

where $N_{k}$ is the density of the radiating species having Einstein coefficient $A_{k}, \sigma_{k m}^{\mathrm{I}}$ is the photo-ionization cross section for species $m$ by photons emitted by species $k$ and $\sigma_{l k}$ is the total absorption cross section for photon $k$ by species $l . G_{k}\left(\vec{r}_{j}^{\prime}, \vec{r}_{i}\right)$ is Green's function for the survival of the photons emitted at the location $\vec{r}_{j}$ to reach location $\vec{r}_{i}$, and also accounts for view angles and obscuration.

Emission of electrons from the dielectric surface results from two processes-secondary electron emission by ion bombardment and photoelectron emission. (The values of the electric fields are not large enough for field emission to be important.) Electron emission by photons striking surfaces was included with probability 0.01 . The UV fluxes striking surface location $\vec{r}_{i}$ are given by

$$
\phi\left(\vec{r}_{i}\right)=A_{k} \int N_{k}\left(\vec{r}_{j}^{\prime}\right) G_{k}\left(\vec{r}_{j}^{\prime}, \vec{r}_{i}\right) \mathrm{d}^{3} \vec{r}_{j}^{\prime} .
$$

In this investigation, the source of photons was emission by $\mathrm{Ne}_{2}^{*}$ with wavelength $85 \mathrm{~nm}$. The rationale was that there is moderately rapid, though not instantaneous, conversion of $\mathrm{Ne}$ atomic excited states to the dimer and the boundfree transition in the dimmer producing this radiation is not resonantly absorbed or trapped. The single photo-ionization process we included was for ground state xenon with cross section $10^{-16} \mathrm{~cm}^{2}$. Although the radiative lifetime of $\mathrm{Ne}_{2}^{*}$ is only $11 \mathrm{~ns}$, the precursor states for forming $\mathrm{Ne}_{2}^{*}$ are metastable (or effectively metastable due to radiation trapping) excited

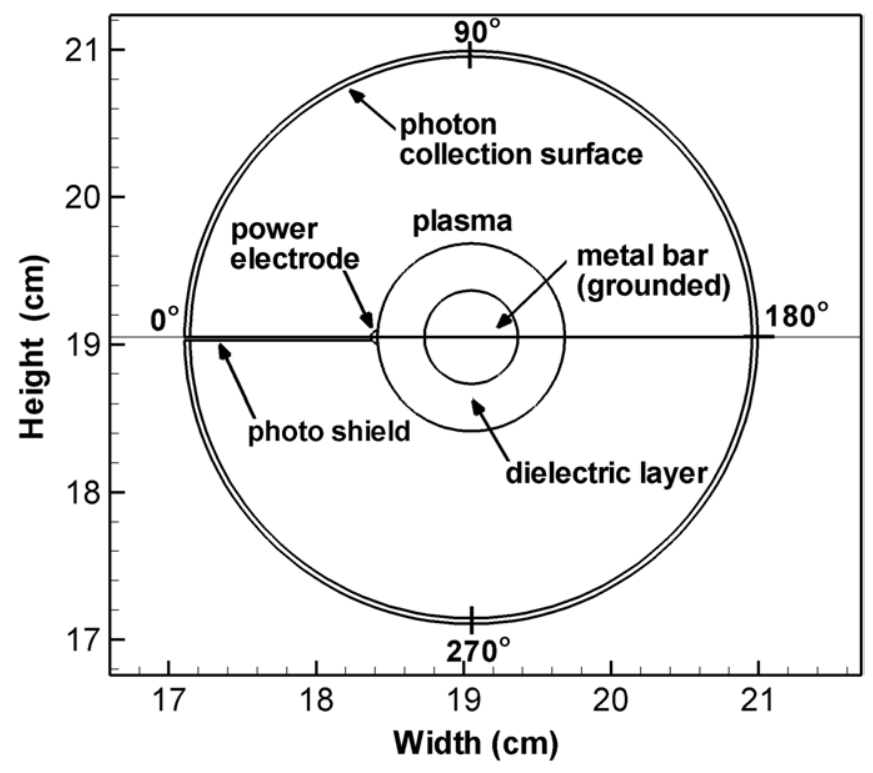

Figure 1. Configuration of the corona-bar corona discharge. The central anode rod is surrounded by a dielectric layer. The photon collecting surface and photon shields are dielectrics. Angles are measured from the high voltage electrode in the clockwise direction. The entire computational domain is a square of $38 \mathrm{~cm}$ on a side with the corona bar located in its centre.

states. As a result, the source of $85 \mathrm{~nm}$ photons can last for times considerably longer than the $\mathrm{Ne}_{2}^{*}$ lifetime. For diagnostic purposes, we also tracked the transport of visible radiation from a short lived, untrapped state of Xe having a lifetime of $5 \mathrm{~ns}$.

The trajectories of secondary electrons emitted from surfaces were followed using a Monte Carlo simulation which is described in detail in [21]. Statistics on the trajectory of the emitted electrons and electrons resulting from their ionizations are collected to compute electron energy distributions (EEDs) as a function of position. These EEDs are used to compute electron impact source functions and sources of secondary electrons slowing to join the bulk electron distribution. These electron impact sources and sources of charge are then included in the continuity equations for charged and neutral species in the fluid portion of the model. A secondary electron emission coefficient of 0.15 was used for all ions unless otherwise noted.

The gas mixture used here is $\mathrm{Ne} / \mathrm{Xe}=99 / 1$ at a constant pressure of $3.5 \mathrm{~atm}$ and temperature of $338 \mathrm{~K}$. This mixture was chosen as being representative of high pressure excimer lasers, albeit in the absence of the attaching gas. A reduced reaction mechanism was formulated that captures the dominant plasma processes without being too computationally burdensome. This reaction mechanism is essentially the same as that which appears in table 1 of [24]. Other than photo-ionization of Xe, the plasma is nearly transparent to the UV light emitted from $\mathrm{Ne}_{2}^{*}$ due to the lack of resonance trapping.

The model geometry consists of a corona bar and a small powered electrode placed on the dielectric surface, as shown in figure 1. The corona bar is an annular dielectric tube around a grounded metal cylinder acting as the anode. The diameter of the anode is $0.63 \mathrm{~cm}$. The outer diameter of the dielectric is $1.27 \mathrm{~cm}$ with dielectric constant $\varepsilon=8$ unless otherwise noted. The powered cathode has a semi-circle shape of width 
$0.5 \mathrm{~mm}$ and is placed on the corona-bar surface. At $t=0$, the voltage on the cathode has a stepwise change to $-25 \mathrm{kV}$ unless noted. The discharge is initiated by placing a small spot of plasma at the intersection of the cathode and corona bar in the upper plane, $3 \times 10^{10} \mathrm{~cm}^{-3}$ in density and $100 \mu \mathrm{m}$ in diameter. The initial electron density is otherwise zero. This initiates a surface discharge in the clockwise direction. The resolution of the numerical mesh was approximately $25 \mu \mathrm{m}$ along the surface of the dielectric.

A circular dielectric surface was placed at a radius of $3.8 \mathrm{~cm}$. The inner surface of this cylinder was used to collect photon fluxes during the discharge. The entire computational domain is a square of $38 \mathrm{~cm}$ on a side with the corona bar located in its centre. A thin dielectric layer is also placed along the horizontal axis between the powered electrode and the photon collector. The purpose of this surface is to prevent photons and charges produced during the initial discharge near the cathode in the upper half plane from crossing over into the lower half plane and initiating a second discharge propagating in the counter-clockwise direction. This enables us to study a single propagating discharge (although an example of counterpropagating discharges will be discussed). To identify the location of the streamer, angles relative to the high voltage electrode will be cited, $0^{\circ}$ at the high voltage electrode and increasing in the clockwise direction, as shown in figure 1.

\section{Propagation of surface discharges}

The characteristics of a corona-bar discharge without the isolating dielectric sheet will first be discussed. Immediately after the application of the voltage pulse, the discharge starts from the area with seed electrons and propagates along the surface of the corona bar in the clockwise direction. As the plasma diffuses to larger radii near the high voltage electrode, photo-ionization produces electrons on the opposite side of the high-voltage electrode, and a second surface discharge is launched in the counter-clockwise direction. The electron temperature, $T_{\mathrm{e}}$, and electric potential, $\Phi$, of the primary and secondary ionization fronts are shown in figure 2 . Since $T_{\mathrm{e}}$ is maximum in the high electric field of the heads of the streamers, it serves as a good diagnostic for the location of the avalanche front. As the counter-propagating streamers follow the coronabar surface, the two ionizing fronts charge the corona bar as shown by the electric potential contours in figure 2 . The two counter-propagating fronts eventually intersect with each other at a location slightly below the horizontal line (due to the time delay of the second discharge). At the site of the collision of the ionization fronts, the equal potential lines from both ends connect and the electric field starts to diminish, and so does $T_{\mathrm{e}}$. As the surface is charged, $T_{\mathrm{e}}$ decreases from an initial value of $7 \mathrm{eV}$ to a final value of $3.8 \mathrm{eV}$ when the plasma channels intersect. The characteristics of the counterclockwise surface discharge are similar to that of the primary clockwise propagating discharge, but just delayed in time due to its later launching.

To simplify the analysis of the surface discharge, the following examples employ the dielectric shield to prevent UV light and charges from crossing to the other side of the
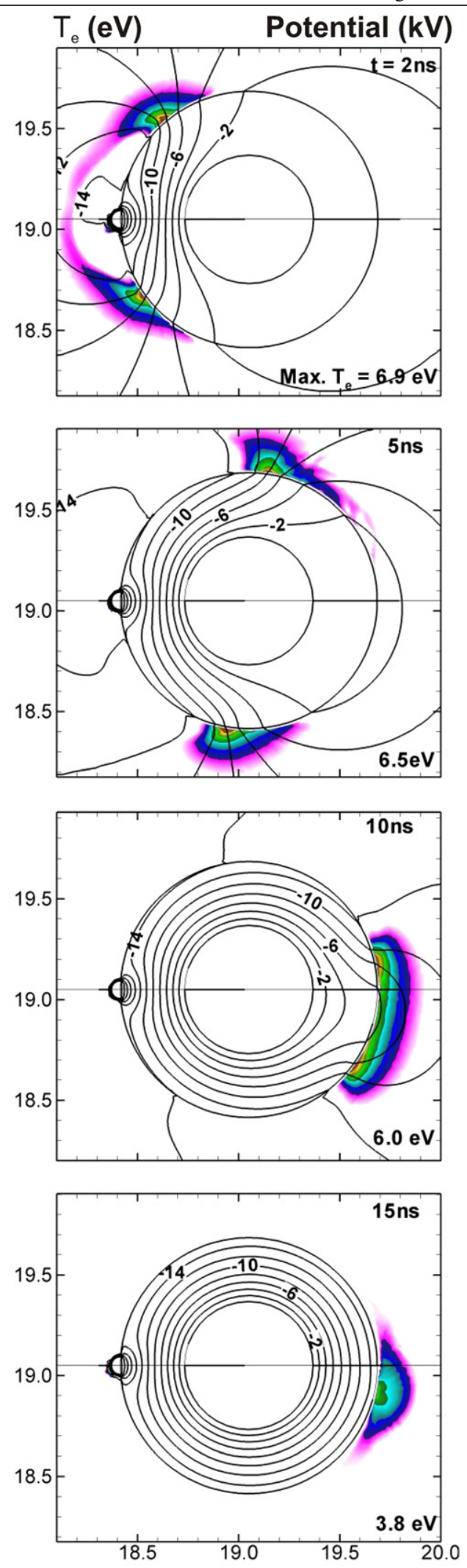

MIN

MAX

Figure 2. Electron temperature $T_{\mathrm{e}}$ (flood) and electric potential $\Phi$ (lines) of two ionization fronts launched from the cathode without the photon shield at progressive times after initiation (at $t=2,5,10$ and $15 \mathrm{~ns}$ ). Potentials are in $\mathrm{kV}$. The maximum value of $T_{\mathrm{e}}$ is noted in each frame. The counter-clockwise discharge lags the initial clockwise discharge. Their intersections are in the lower plane. 

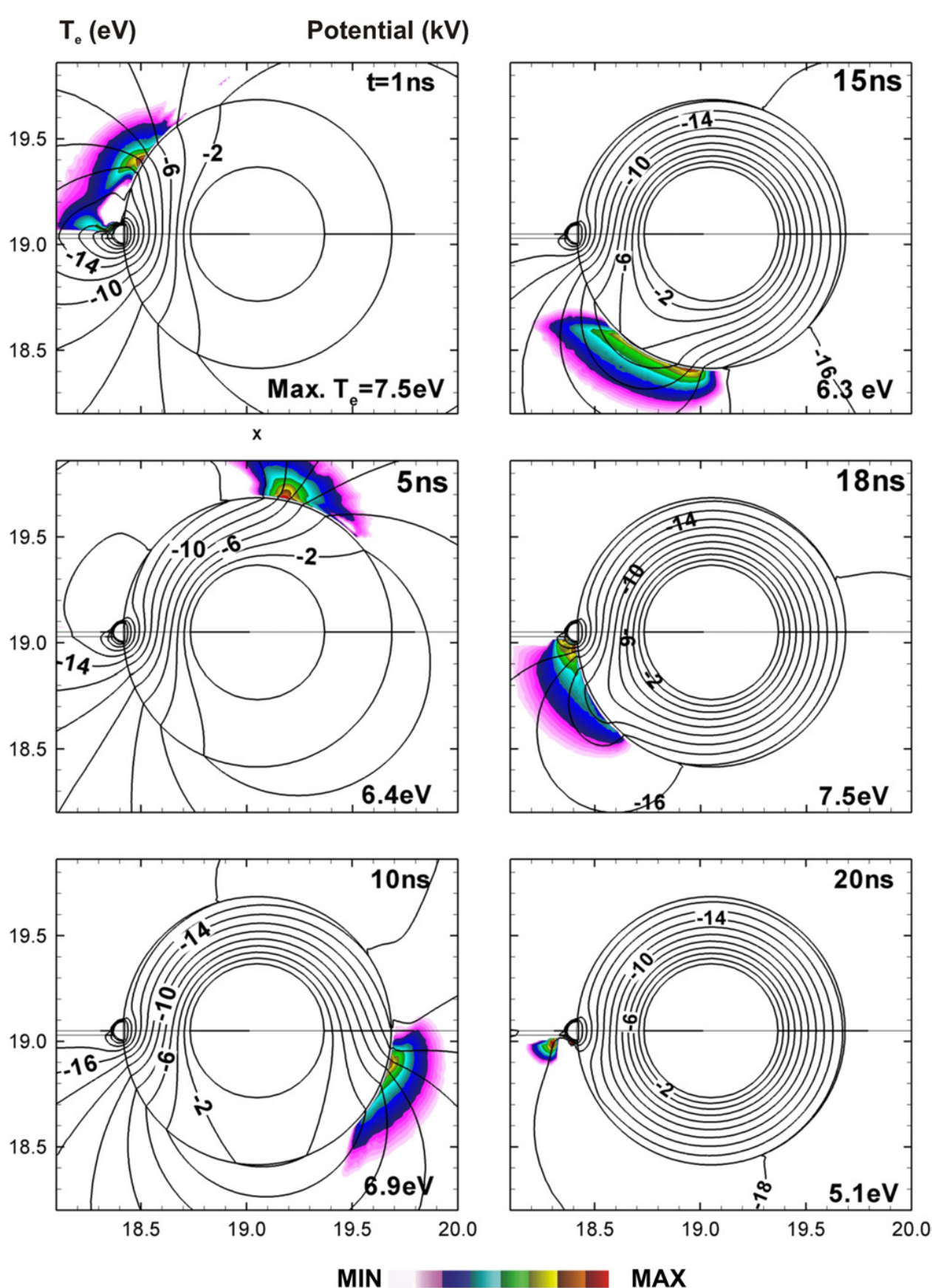

Figure 3. Electron temperature $T_{\mathrm{e}}$ (flood) and electric potential $\Phi$ (lines) of a single discharge (with photon shield in place) at $t=1,5,10$, 15,18 and $20 \mathrm{~ns}$ after initiation. Potentials are in $\mathrm{kV}$. The maximum value of $T_{\mathrm{e}}$ is noted in each frame. As the avalanche propagates along the surface, the dielectric is charged, capturing potential within the solid.

electrode to launch a counter-clockwise surface discharge. For example, $T_{\mathrm{e}}$ and $\Phi$ of a single discharge as it propagates in the clockwise direction over the entire circumference of the corona bar are shown in figure 3. The discharge is driven by a finite component of the electric field along the surface of the dielectric. Similar to a streamer in gas, the discharge propagates by virtue of charge separation at the head of the plasma, which produces an electric field of 50-70 $\mathrm{Td}(1 \mathrm{Td}=$ $10^{-17} \mathrm{~V} \mathrm{~cm}^{2}$ ) and sustains a $T_{\mathrm{e}}$ of up to $7 \mathrm{eV}$. Ionization in this high electric field avalanches the gas, producing a conductive channel with a reduced electric field, thereby advancing the plasma sheet along the surface of the discharge. In addition to these streamer-like properties, electrons from the plasma sheet negatively charge the surface of the dielectric, thereby confining the electric potential to the interior of the corona bar-that is, charging the capacitance of the corona bar.

The average speed of the avalanche front is about $2 \times 10^{8} \mathrm{~cm} \mathrm{~s}^{-1}$, commensurate with that of streamers in the gas phase. The speed of the avalanche front slows as it propagates around the corona bar, from an initial value of $2.5 \times 10^{8} \mathrm{~cm} \mathrm{~s}^{-1}$ for $t<5 \mathrm{~ns}$ to about $1.8 \times 10^{8} \mathrm{~cm} \mathrm{~s}^{-1}$ for $t>15 \mathrm{~ns}$. This slowing of the streamer results from more electric potential being confined within the dielectric as the surface discharge charges the dielectric. This trend is the opposite to that of 
gas phase streamers in DBD discharges where the speed of the avalanche front generally increases as the streamer approaches the dielectric barrier. This latter trend in the gas phase results from the applied voltage being compressed into the nonionized space ahead of the conductive streamer channel, which increases the electric field for subsequent avalanche. In the case of the corona bar, the compression of the voltage occurs within the dielectric, and so that potential is not available for subsequent avalanche. On the other hand, in long, unconfined corona streamers, the speed of the streamer tends to decrease with time as more voltage is dropped across the conductive plasma channel trailing the avalanche front.

When the clockwise discharge rotates to about $320^{\circ}$ UV illumination from the approaching ionization front seeds electrons near the high voltage electrode on the bottom side of the photon shield which then launches a short counterclockwise discharge. The two discharges then quickly intersect and merge at about $340^{\circ}(t=18 \mathrm{~ns})$ which reduces the electric field and $T_{\mathrm{e}}$. After intersection of the counter-propagating discharge fronts, the capacitance of the corona bar is essentially fully charged and the equipotential lines are nearly parallel to the surface, as at $t=20 \mathrm{~ns}$. At this point electron impact ionization largely ceases. The charging of the surface of the dielectric leaves the surface-hugging plasma electropositive. Following the cessation of the surface discharge, diffusion of positive ions from the plasma sheet to the surface neutralizes the negative surface charge thereby discharging the capacitance of the corona bar. With this discharging, the electric potential lines again cross through the surface into the gas.

The electron density $\left(n_{\mathrm{e}}\right)$, electron impact ionization source at the ionizing front with the corresponding $\Phi$ and $T_{\mathrm{e}}$ are shown in figure 4 . Images are shown at $135^{\circ}, 225^{\circ}$ and $315^{\circ}$, rotated to the horizontal. The structure of the ionization front remains qualitatively unchanged as it propagates around the circumference. The electron densities at the leading edge of the avalanche and the trailing sheet of plasma are $5 \times 10^{14}-10^{15} \mathrm{~cm}^{-3}$. The thickness of the plasma sheet is about $200 \mu \mathrm{m}$, which is a factor of 2-3 thicker than the analogous streamer in the gas phase. The electron impact ionization source has its peak at the front, but slightly lags behind the peak of $T_{\mathrm{e}}$ as $E / N$ peaks ahead of the avalanche front. Electron sources are shown in figure 5 at $90^{\circ}$ resulting from bulk electron impact ionization, additional ionization by the secondary electrons through sheath acceleration, secondary electron emission from the surface due to ion and photo fluxes and direct photo-ionization of Xe. The bulk electron impact ionization, of order $10^{23} \mathrm{~cm}^{-3} \mathrm{~s}^{-1}$, has the highest contribution and dominates the avalanche process. The instantaneous ionization from the sheath-accelerated secondary electrons is of order $10^{22} \mathrm{~cm}^{-3} \mathrm{~s}^{-1}$ while the electrons from direct surface secondary emission are another order of magnitude lower. The photo-ionization has the least contribution to the production of the electrons, about $10^{20} \mathrm{~cm}^{-3} \mathrm{~s}^{-1}$. Although this source is small, its spatial distribution extends $0.1 \mathrm{~mm}$ ahead of the avalanche front, thereby seeding electrons to enable propagation of the discharge.

The sheath-accelerated secondary electrons may also produce ionization ahead of the ionization front to aid in its

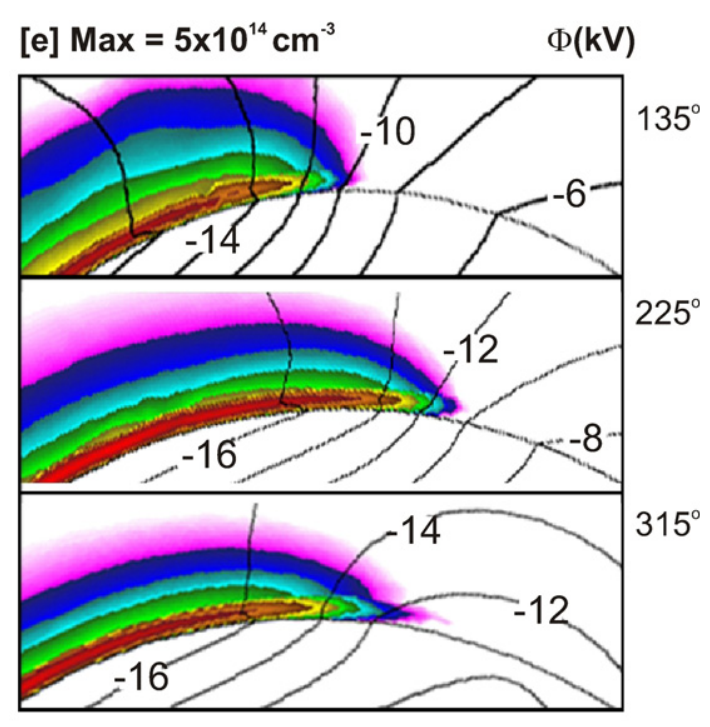

(a)

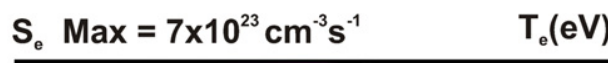

(b)

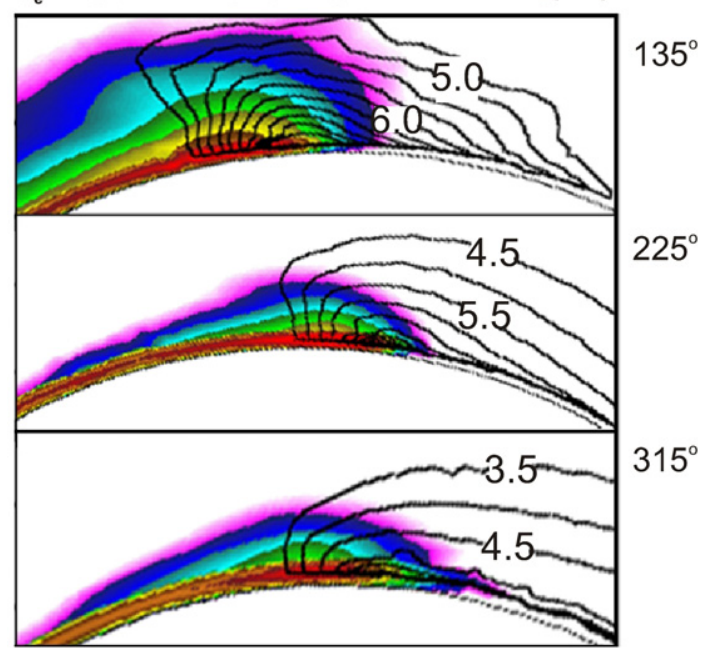

MIN

MAX

Figure 4. The structure of ionization front at azimuthal locations $135^{\circ}, 225^{\circ}, 315^{\circ}$ (top to bottom) relative to the powered electrode. (a) Electron density (flood) and $\Phi$ (lines); and (b) electron impact ionization source (flood) and $T_{\mathrm{e}}$ (lines). Images are rotated for upright alignment. Potentials are in $\mathrm{kV}$. The maximum values are noted in each figure. Densities and sources are plotted on a log scale over 3 decades. The structure of the ionization front does not significantly change along the circumference.

propagation. The ionization sources shown in figure 5(b) show a few ionization tracks due to individual particles in the Monte Carlo simulation that extend ahead of the ionization front. Although the discreteness of the simulation exaggerates this effect, the mean free path for the sheath-accelerated electrons, even at above atmospheric pressure, can be greater than the width of the avalanche front. Secondary electrons emitted from the surface near the head of the streamer will experience an electric field having a component parallel to the surface. This accelerates secondary electrons ahead of the streamer where they produce ionization. A similar phenomenon occurs in the propagation of gas phase streamers [22,23].

One of the estimated parameters in the model is the effective secondary electron emission coefficient, $\gamma$ for ions 


\section{Bulk}

(a) 19.6

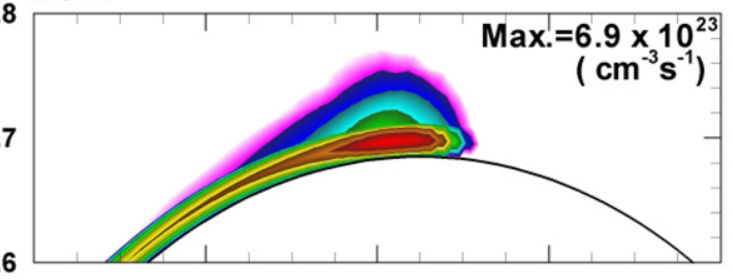

(b)

\section{Secondary electron ionization}

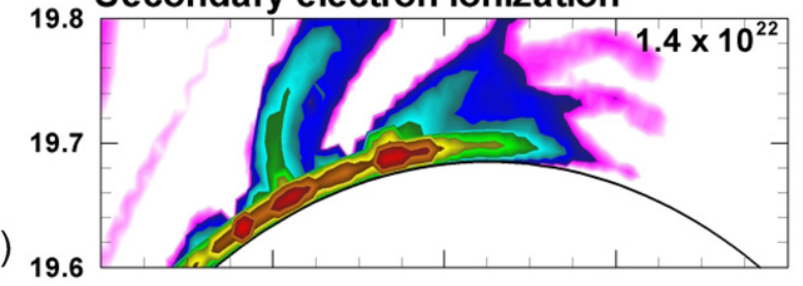

(c)
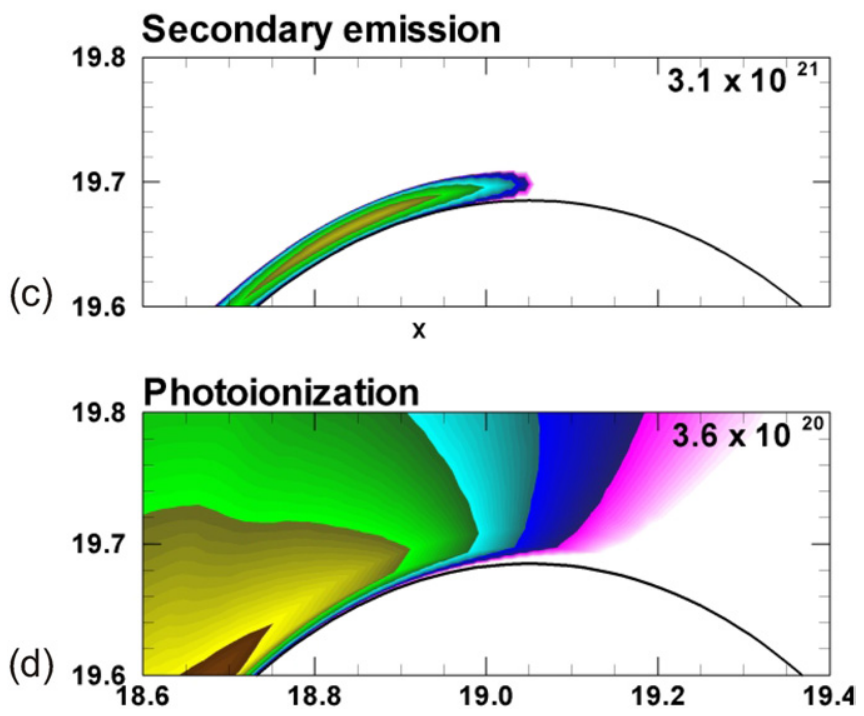

Figure 5. Contributions to ionization at the front from $(a)$ bulk electron impact process, $(b)$ sheath accelerated secondary electrons, (c) direct surface secondary emission due to ion and photon fluxes and $(d)$ photo-ionization of Xe. Sources are plotted on a log scale over 3 decades with the maximum noted in each frame. Ionization by bulk electrons dominates though photo-ionization and ionization by secondary electrons is over a more extended volume. Secondary electron ionization tracks due to discrete Monte Carlo particles are exaggerated due to the discreteness of the calculation, but nevertheless indicate the possible range of secondary electrons.

on the dielectric. To gauge the importance of this estimate, simulations were performed with different values of $\gamma$ ranging from 0.05 to 0.20 and the results are shown in figure 6 . The contribution to ionization from the sheath-accelerated secondary electrons increases by about $50 \%$ when $\gamma$ increases from 0.05 to 0.20 . The contribution from bulk electron impact ionization increases by about $5 \%$ for this range (based on maximum values). It remains the dominant source of ionization, being larger than ionization by sheath-accelerated electrons by a factor of about 30 . The combined effects of increasing $\gamma$ over this range produces an increase in the maximum electron density of about $16 \%$ and an increase in speed of the avalanche front about 3\%. Uncertainties in $\gamma$ affect the details of the propagation of the ionization front but the structure of the discharge is not strongly affected.
The distributions of $\Phi, \mathrm{E}, T_{\mathrm{e}}$ and $n_{\mathrm{e}}$ along a circumference about $50 \mu \mathrm{m}$ above the surface are shown in figure 7 for times up to $20 \mathrm{~ns}$. The noise in the plots results from the statistics of the electron Monte Carlo simulation and from interpolation from the unstructured mesh onto the circumference. A progressive decrease in the potential occurs at the ionization front as the dielectric is charged which then removes voltage from streamer. At $20 \mathrm{~ns}$ the charging of the dielectric is nearly complete. $T_{\mathrm{e}}$ peaks in the large electric field of the ionization front, and remains above $2-3 \mathrm{eV}$ in the ionized layer above the dielectric behind the ionization front. This is qualitatively a higher $T_{\mathrm{e}}$ than in the ionized channel of the analogous gas phase streamer, a consequence of the charged particle loss to the surface which increases the resistance of the plasma layer. The propagation speed is high at the initial stage, at around $2.5 \times 10^{8} \mathrm{~cm} \mathrm{~s}^{-1}$, but decreases with time and distance, particularly after $15 \mathrm{~ns}$. The variation of electric fields is non-monotonic, with higher values at $10 \mathrm{~ns}$ than at 5 and $15 \mathrm{~ns}$. This is due to the combined effects of surface charging and the geometric curvature of the surface. Based on only the geometry, the electric fields are maximum adjacent to and on either side the high voltage electrode. As the surface is charged, the potential contours are captured within the dielectric, translating the gradient in the potential further along the circumference. At the same time, the voltage drop available along the surface is being reduced due to the potential being captured in the dielectric. The combination of these effects is that there is a small increase in electric field to about $45 \mathrm{kV} \mathrm{cm}^{-1}(53 \mathrm{Td})$ at $10 \mathrm{~ns}$ at $190^{\circ}$. As the ionization front continues to move into the lower half plane back towards the cathode, the electric field strength starts to decrease again. This is at a time when the counter-clockwise discharge has started to propagate, charging the dielectric as it moves. At $20 \mathrm{~ns}$ when the two discharges intersect, the dielectric has been essentially fully charged, and the electric field rapidly decays.

At $3.5 \mathrm{~atm}$, even on these short time scales, the electron collision frequency is high enough that $T_{\mathrm{e}}$ is in near equilibrium with the electric field. The time and spatial history of $T_{\mathrm{e}}$ then closely follows that of the electric field. The progressively longer extension in $T_{\mathrm{e}}$ in front of the leading edge of the ionization front results directly from the distribution of the electric field at its leading edge. The electron density shows a steep rise at the ionization front and continues to increase with time once the front passes. The peak electron density at $t=1 \mathrm{~ns}$ is around $2 \times 10^{14} \mathrm{~cm}^{-3}$ but increases by one order of magnitude at $t>5 \mathrm{~ns}$. At $t=20 \mathrm{~ns}, n_{\mathrm{e}}$ has a minimum around $3.6 \mathrm{~cm}$, which results from the intersection between the primary and secondary discharges.

One of the characteristics of surface discharges is the large gradient of the electric potential normal to the surface. In a gas phase streamer, the electric field is dominant in the direction of propagation while the electric field perpendicular to the direction of propagation largely results from ambipolar forces. In surface discharges, there are vacuum field components of the electric field perpendicular to the surface and the direction of propagation in addition to those resulting from ambipolar forces. Consequently, the plasma in the surface discharge is typically thicker than its gas phase analogue. The continuous 


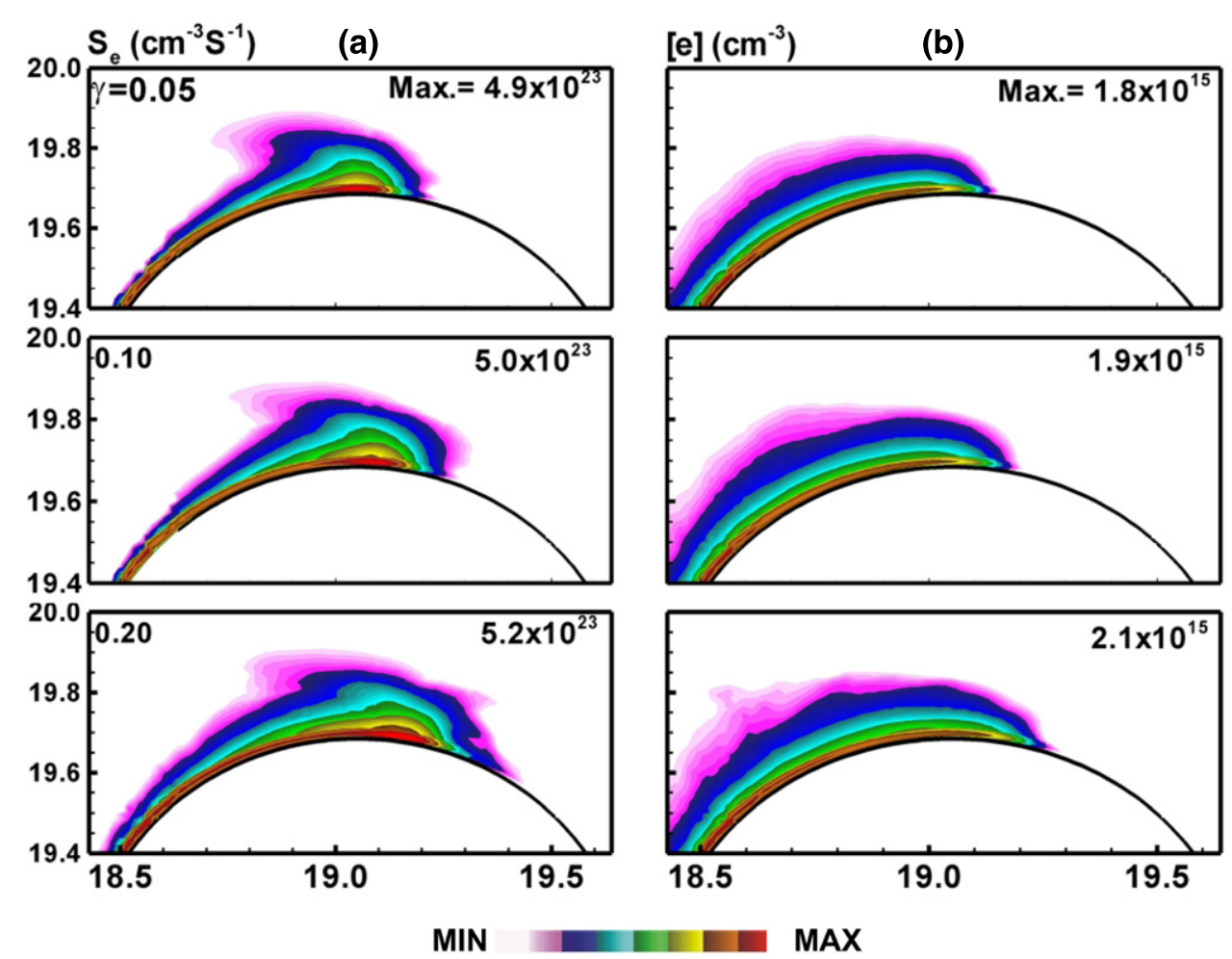

Figure 6. Consequences of secondary electron emission coefficient by ions, $\gamma=0.05-0.20$ on plasma properties at $t=5 \mathrm{~ns}$. ( $a$ ) Ionization sources from electron impact by bulk electrons (the dominant ionization source) and $(b)$ electron density. The ionization source and electron density increase by $5-15 \%$ over this range of $\gamma$ (based on maximum values.)

breakdown of the gas takes place predominately within the sheet although electron diffusion and photo-ionization take place over a more extended area.

The electron density and electric field in the ionization front are shown in figure 8 at $t=4.8 \mathrm{~ns}\left(90^{\circ}\right)$. The electric field has a peak of about $40 \mathrm{kV} \mathrm{cm}^{-1}(\approx 47 \mathrm{Td})$ close to the surface, from which it spreads in both normal and tangential directions. This forms a sharp leading edge and a blunt trailing edge. The leading edge of the electron density coincides with the peak of the electric field, so the electron avalanche occurs predominately in the trailing edge of the high field. Ahead of the ionization front, the normal component $E_{\mathrm{n}}$ is positive which drives electrons drifting towards the surface. Once crossing the front, the sign of $E_{\mathrm{n}}$ reverses as a result of the charging of the surface by the plasma. The tangential component $E_{\mathrm{t}}$ remains negative across the ionization front but peaks at the leading edge of the electron density where the space charge is maximum. This large $E_{\mathrm{t}}$ enables electrons to drift from the plasma region behind the front into non-ionized gas ahead of the front and thus serve as seeding electrons for further avalanche.

Typical discharge propagation times are tens of ns, and this is much shorter than the acoustic time scale of the neutral gasthat is, the gas does not have time to get significantly heated, move and rarefy during the current pulse. Gas dynamics effects are therefore insignificant during the current pulse. For example, for otherwise identical conditions, simulations were performed with and without solving the compressible NavierStokes equations. Gas temperatures at the ionization front increased by at most a few degrees.
Electron and ion densities at a fixed point located at $270^{\circ}$ and $100 \mu \mathrm{m}$ above the dielectric surface are shown in figure $9(a)$. The electron and $\mathrm{Xe}^{+}$densities have nearly identical sharp rises to a maximum of $2.0 \times 10^{15} \mathrm{~cm}^{-3}$, showing the ionization occurs first through Xe due to its lower ionization potential. After the ionization front, $n_{\mathrm{e}}$ decays slowly due to dissociative recombination with $\mathrm{Xe}_{2}^{+}$and $\mathrm{Ne}_{2}^{+}$which is on the time scale of tens of ns. The $\mathrm{Ne}^{+}$density has a peak of $2.0 \times 10^{13} \mathrm{~cm}^{-3}$. After the avalanche front passes, the $\mathrm{Ne}^{+}$ density quickly decreases with time while the densities of molecular ions $\mathrm{Xe}_{2}^{+}$and $\mathrm{Ne}_{2}^{+}$increase as a result of 3-body association reactions. Since $T_{\mathrm{e}}$ in the plasma channel, $2 \mathrm{eV}$, is below that which directly ionizes $\mathrm{Ne}$, the $\mathrm{Ne}^{+}$density is not replenished. The $\mathrm{Xe}^{+}$density is sustained by some continuing electron impact ionization and Penning ionization from $\mathrm{Ne}$ excited states.

The densities of atomic and molecular excited states at the same point are shown in figure $9(b)$. As the ionization front passes, both $\mathrm{Ne}^{*}[\mathrm{Ne}(3 \mathrm{~s})]$ and $\mathrm{Xe}^{*}[\mathrm{Xe}(6 \mathrm{~s})]$ are produced by direct electron impact and thus their densities first rise sharply but fall once $T_{\mathrm{e}}$ decreases. After the passage of the ionization front, the density of $\mathrm{Xe}(6 \mathrm{~s})$ first decreases and then achieves a nearly constant value, a quasi-balance between sources due to dissociative recombination of $\mathrm{Xe}_{2}^{+}$and losses due to formation of $\mathrm{Xe}_{2}^{*}$. The density of $\mathrm{Ne}(3 \mathrm{~s})$ continues to decrease due to the low $T_{\mathrm{e}}$ which fails to replenish its density. After a slower rise compared with the atomic excited species, the density of $\mathrm{Xe}_{2}^{*}$ also nearly reaches a constant value. The density of the VUV emitting species $\mathrm{Ne}_{2}^{*}$ has a maximum of $5 \times 10^{11} \mathrm{~cm}^{-3}$. Although the radiative lifetime of $\mathrm{Ne}_{2}^{*}$ is $11 \mathrm{~ns}$, it is formed by 

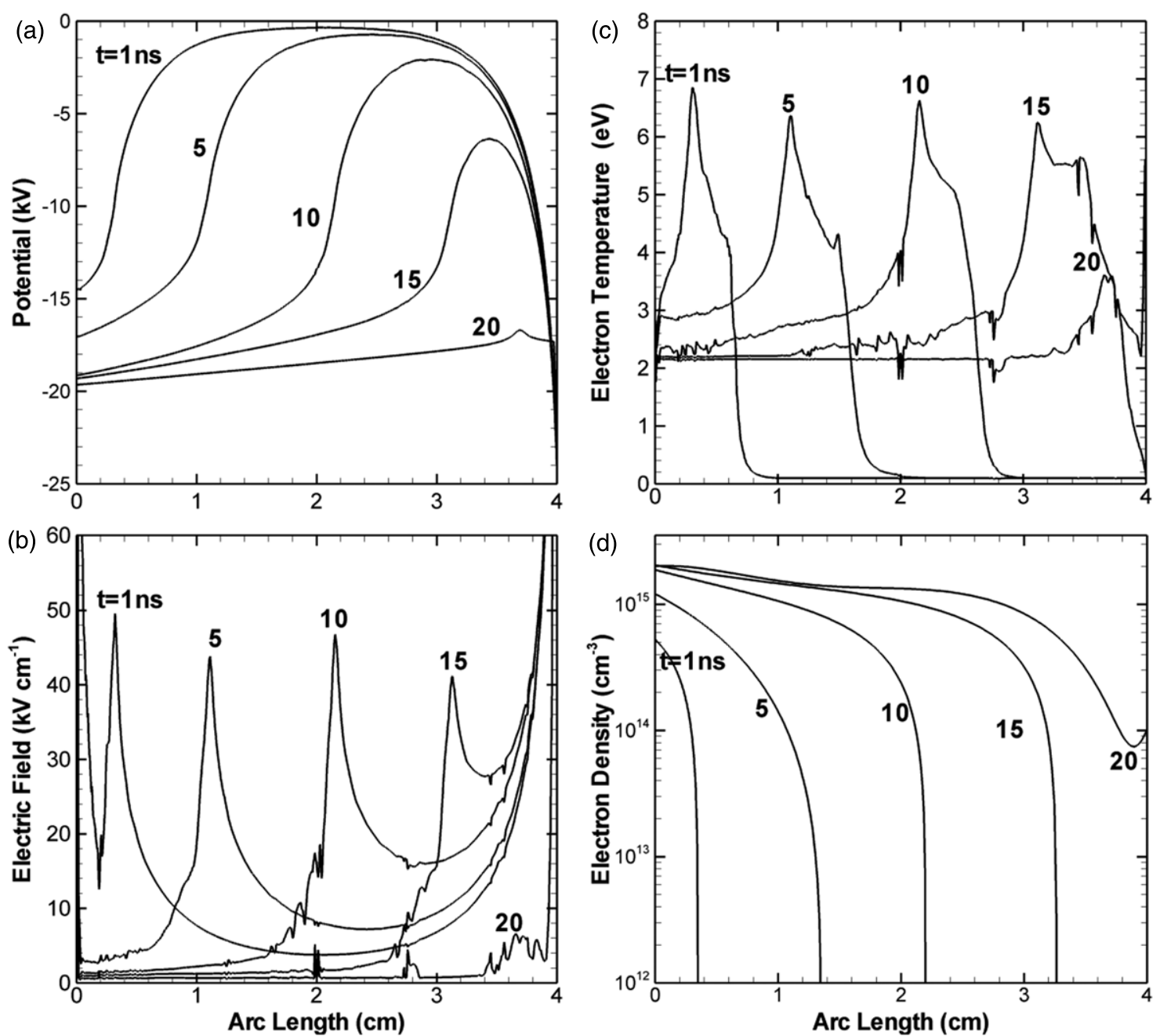

Figure 7. Circumferential distribution of $(a)$ electric potential, $(b)$ electrical field, $(c)$ electron temperature and $(d)$ electron density at $t=1$, $5,10,15$ and $20 \mathrm{~ns}$. The arc length of $0-4 \mathrm{~cm}$ maps onto $0^{\circ}-360^{\circ}$ beginning at the cathode. The electric field has non-monotonic behaviour along the circumference due to the combined effects of geometry and surface charging.

3-body association reaction with $\mathrm{Ne}(3 \mathrm{~s})$, which has a longer persistence. So, compared with the traversal time of the ionization front, the effective lifetime of the $\mathrm{Ne}_{2}^{*}$ is relatively long.

\section{Photon fluxes}

Due to the lack of the resonance absorption, the VUV light emitted from $\mathrm{Ne}_{2}^{*}$ during the corona discharge experiences little absorption by other than Xe when passing through the plasma. The photon fluxes incident on the collector surface at different times as a function of position $0^{\circ}$ to $360^{\circ}$ (corresponding to an arc length of $0-12 \mathrm{~cm}$ ) are shown in figure $10(a)$. As the ionization front traverses the corona-bar surface, the collector is progressively illuminated according to the distribution of $\mathrm{Ne}_{2}^{*}$ and the view angle spanned by the collector surface. The fluxes are $3 \times 10^{16} \mathrm{~cm}^{-2} \mathrm{~s}^{-1}$ to $2 \times 10^{17} \mathrm{~cm}^{-2} \mathrm{~s}^{-1}$. Initially, the VUV light production lags the ionization front but increases rapidly up to $10 \mathrm{~ns}$ when the ionization front is at about half way around the circumference. This is in large part a result of the collector at any given point integrating emission received from $\mathrm{Ne}_{2}^{*}$ distributed on nearly half the circumference of the corona bar. While the ionizing front continues to traverse the corona bar and create new emitters, the photon fluxes emitted from the surface behind the front start to decay. This causes the peak of the photon flux on the collecting surface to shift gradually forward. By $t=20 \mathrm{~ns}$, the peak flux has moved into the lower half of the collector surface. Once the ionization front has traversed the surface, the photon fluxes decay monotonically with time but maintain a relatively uniform circumferential profile.

To illustrate the effect of a lifetime of emitting species on the photon flux distribution, we tracked emission from an optical transition from $\mathrm{Xe}(6 \mathrm{p})$ with an artificially short lifetime of $5 \mathrm{~ns}$. The photon flux distribution on the collector surface from this transition is shown in figure $10(b)$. The peak magnitude of the photon flux is nearly three orders of magnitude higher than that from $\mathrm{Ne}_{2}^{*}$, due in large part to the more rapid rate of population of the lower energy levels of $\mathrm{Xe}$ and shorter lifetime. However, the decay of the photon flux after the passing of the ionization front is also more rapid due to the shorter lifetime of the state. For example, at $t=5 \mathrm{~ns}$ the peak of the photon flux has moved further from the cathode region as compared with the flux from $\mathrm{Ne}_{2}^{*}$. Between 5 and $20 \mathrm{~ns}$, the photon collector is progressively illuminated 

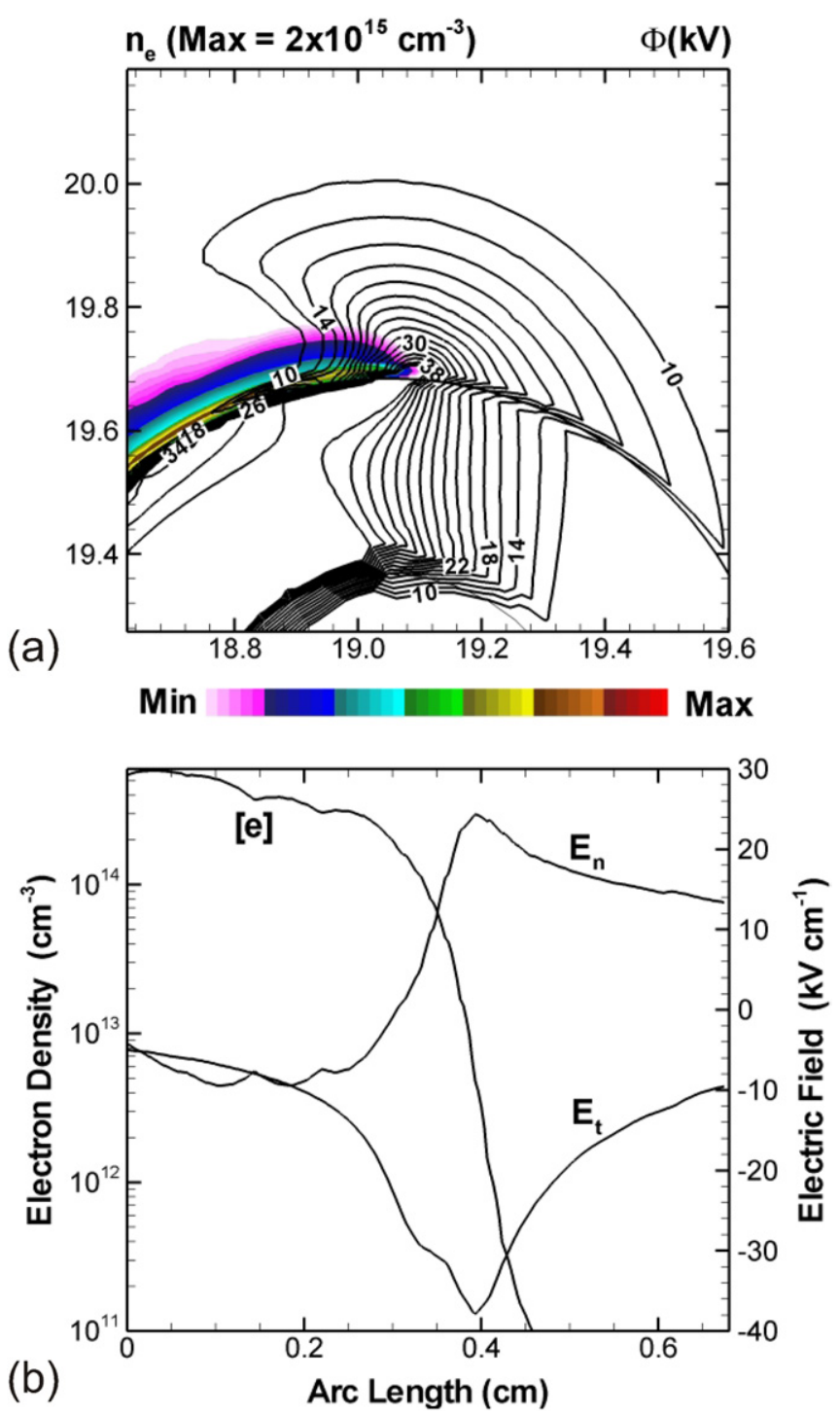

Figure 8. Structure of the ionization front. (a) Electron density (flood) and electric field $E$ (lines); and $(b)$ electron density, normal $\left(E_{\mathrm{n}}\right)$ and tangential $\left(E_{\mathrm{t}}\right)$ electric fields cross the ionization front. The normal electric field switches sign across the ionization front.

following the motion of the avalanche front. With direct electron impact being the primary source of $\mathrm{Xe}(6 \mathrm{p})$, the peaks of the photon flux in this case remain in the upper portion of the circumference, following from the higher electron temperature in that region.

\section{Dielectric constant and applied voltage}

One of the key design decisions for the pre-ionizer is the dielectric constant of the corona-bar material. The larger $\varepsilon / \varepsilon_{0}$, the larger the capacitance of the corona bar and so more charge can be stored on its surface. The length of the plasma channel and $T_{\mathrm{e}}$ in the ionization front as it traverses the circumference of the corona bar are shown in figure 11 for dielectric constants of $\varepsilon / \varepsilon_{0}=2$ to 32. The propagation speed of the ionization front (given by the slope of the line in figure $11(a)$ ) decreases with time but with the exception of $\varepsilon / \varepsilon_{0}=2$, the deceleration is not particularly large. The

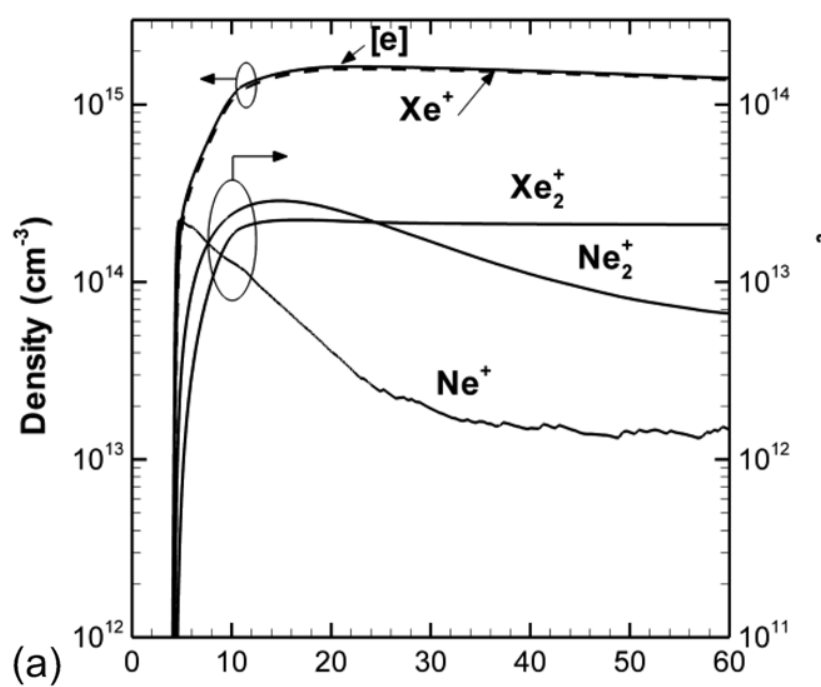

?ำ

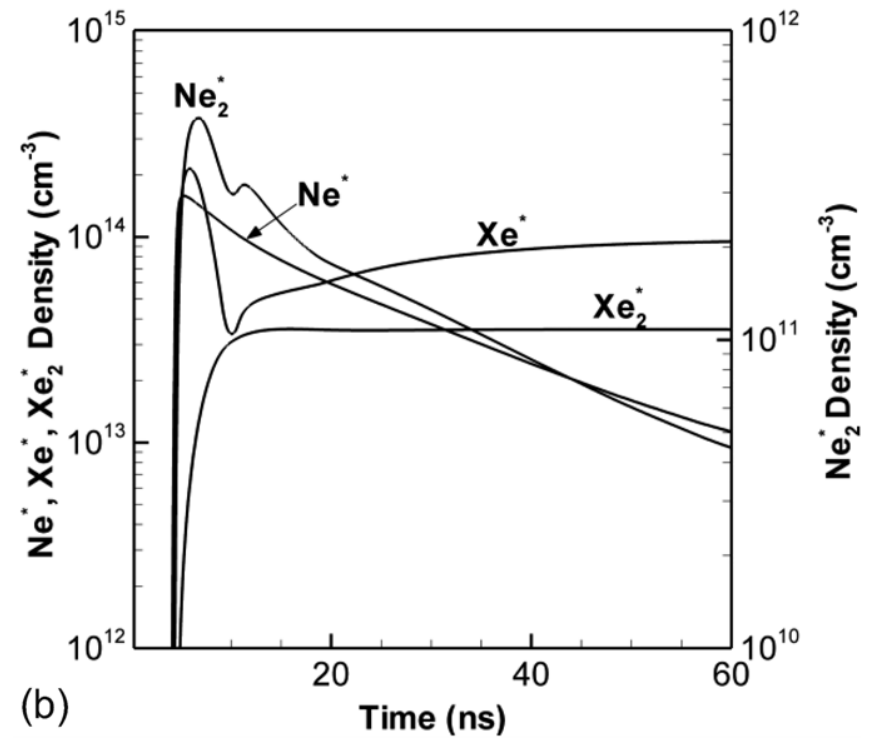

Figure 9. Densities as a function of time at $270^{\circ}$ along the circumference and $100 \mu \mathrm{m}$ above the dielectric surface. (a) Electron and positive ions and $(b)$ neutral excited states. The Ne species are dominantly produced by the transit of the ionization front.

maximum speed is about $3.0 \times 10^{8} \mathrm{~cm} \mathrm{~s}^{-1}$ for $\varepsilon / \varepsilon_{0}=2$ at the start of the avalanche. For $t<5 \mathrm{~ns}$, which corresponds to the first quarter of the circumference, the propagation speed decreases with increasing dielectric constant. This is likely due to the increased capacitance of the corona bar. The streamer needs to spend more time above the surface to fully charge it. However, it is also found that the electron density of the streamer increases with increasing dielectric constant, which partially offsets the capacitance effect and reduces the difference in the propagation speeds. These observations are consistent with predications for a sliding discharge on a flat surface [25]. As the ionization front moves further along the circumference the dependence of the propagation speed on dielectric constant is not necessarily monotonic due to the complex electric field structure and surface curvature. The corresponding $T_{\mathrm{e}}$ at the moving ionization front shows this more clearly. After a quick decay up to about $5 \mathrm{~ns}, T_{\mathrm{e}}$ increases 
(a)
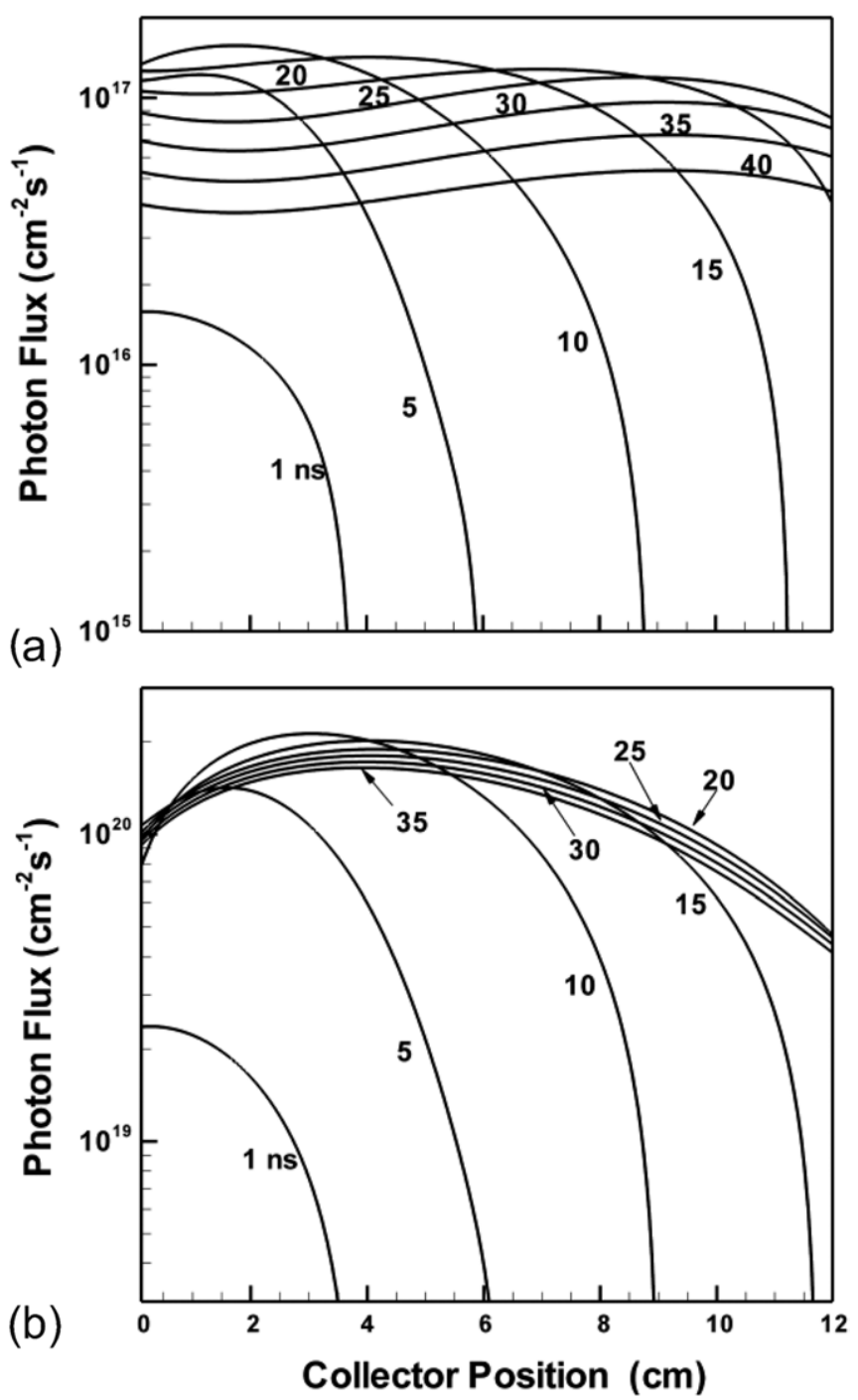

Figure 10. Photon fluxes on the collector surface for different times. (a) VUV from $\mathrm{Ne}_{2}^{*}$ and $(b)$ visible radiation from a short lived state of Xe.

for a period of time before a final decay. Within this period, the peak $T_{\mathrm{e}}$ increases with increasing dielectric constant.

For a fixed dielectric constant of $\varepsilon / \varepsilon_{0}=8$ the traversal distance and $T_{\mathrm{e}}$ of the discharge front are shown in figure 12 for applied voltages of $V=-10$ to $-30 \mathrm{kV}$. The propagation speed increases with increasing voltage up to $3.5 \times 10^{8} \mathrm{~cm} \mathrm{~s}^{-1}$, nearly 12 times of that at $-10 \mathrm{kV}$. The peak $T_{\mathrm{e}}$ (after the intital decay) also increases with increasing voltage. However, the structure and propagation characteristics of the ionization front at these different voltages are found to be qualitatively similar, and the non-monotonic variation of $T_{\mathrm{e}}$ is present at all the voltages.

\section{Concluding remarks}

Corona bar, surface discharges are often used as sources of UV/VUV radiation. Using results from a two-dimensional plasma hydrodynamics model with radiation photon transport, we have discussed characteristics of surface discharges in a $3.5 \mathrm{~atm} \mathrm{Ne} / \mathrm{Xe}=99 / 1$ gas mixture. After the initial
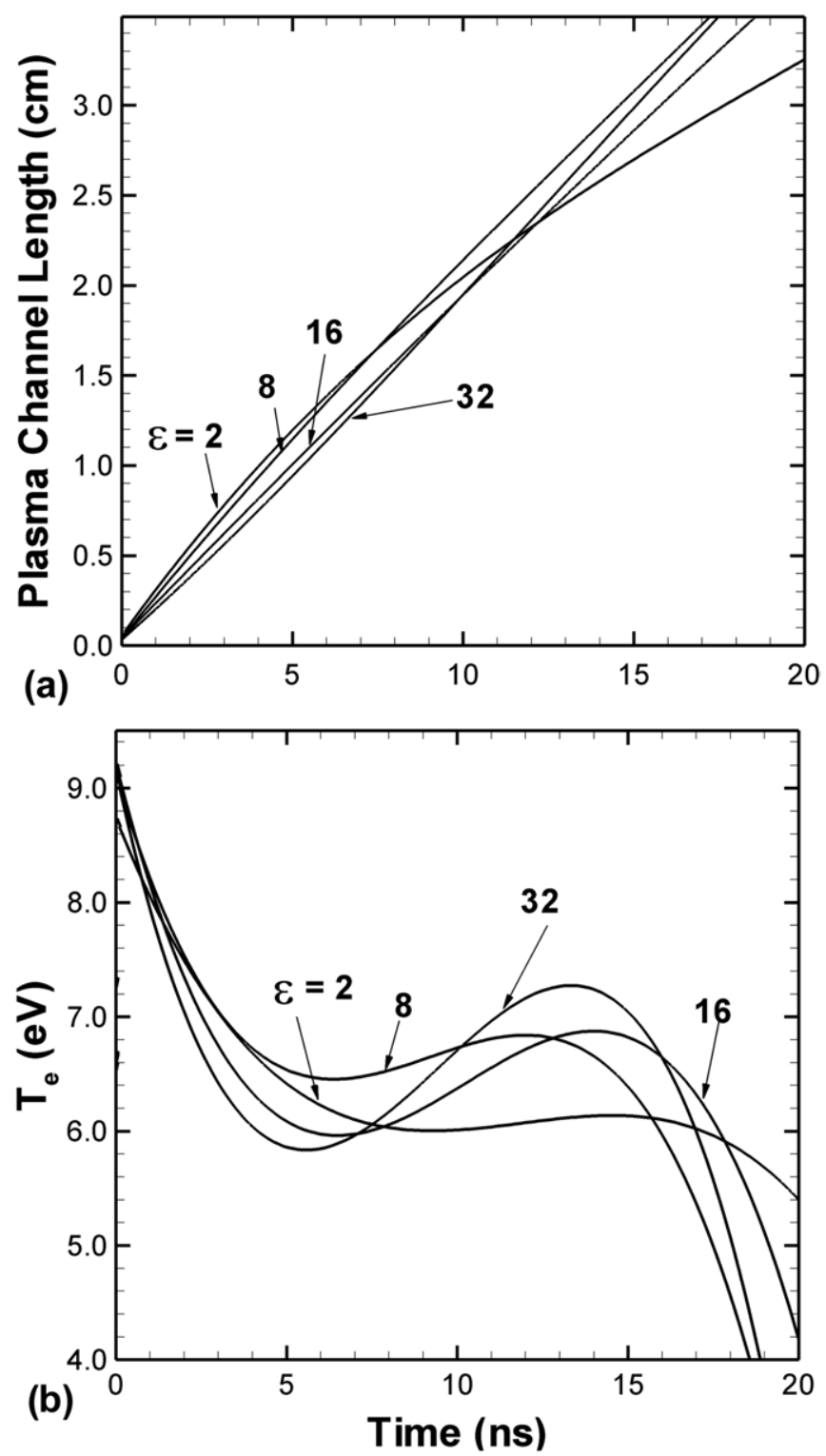

Figure 11. Surface discharge properties at the ionization front as a function of time for different dielectric constants. ( $a$ ) Length of plasma channel and $(b)$ electron temperature. Although initially the streamer propagates more quickly with lower dielectric constant, propagation speeds are not monotonic.

gas breakdown near the high-voltage electrode, an ionization front propagates along the circumference and produces a thin plasma sheet (100-200 $\mu \mathrm{m}$ thick) close to the dielectric surface. The propagation speed decreases with traversal distance, and depending on the dielectric constant and applied voltage, the maximum speed is up to $3.5 \times 10^{8} \mathrm{~cm} \mathrm{~s}^{-1}$. The ionization front is produced by a moving high-field region with sharp leading edge and blunt trailing edge where most of the ionization occurs. Across the avalanche front, the normal component of the electric field changes sign while the tangential field reaches a maximum. This electrical structure enables electrons to drift across the front and produce further avalanche ahead of it. The ionization is produced primarily through bulk electron impact, with the contributions from sheath-accelerated secondary electrons and photo-ionization 

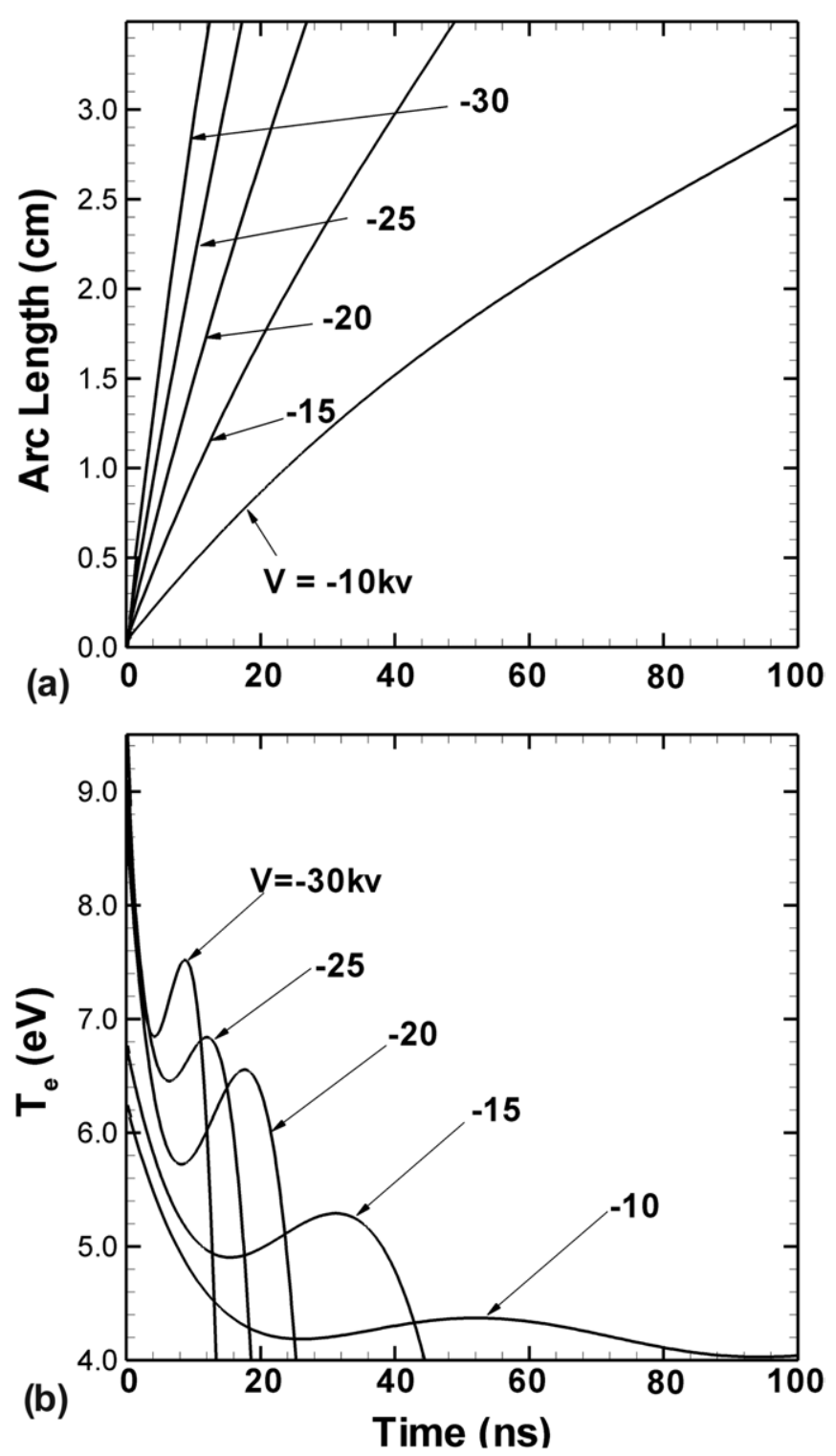

Figure 12. Surface discharge properties at the ionization front as a function of time for different cathode voltages. (a) Length of plasma channel and $(b)$ electron temperature. The speed of propagation and electron temperature increase with increasing voltage.

being one to three orders of magnitude lower. However, photoionization and production of secondary electrons from the surface play roles in propagation of the discharge front. With a combined effect of surface charge deposition and geometric curvature, the magnitude of the electric field and the associated $T_{\mathrm{e}}$ may vary in a non-monotonic manner as the ionization front traverses the circumference. The VUV light fluxes were collected on a circular surrounding surface. Their distributions are correlated with the moving ionization front but with a time delay due to the relative long lifetime of the precursor to the emitting species $\mathrm{Ne}_{2}^{*}$.

\section{Acknowledgment}

This work was supported by Cymer, Inc., and the Department of Energy Office of Fusion Energy Sciences.

\section{References}

[1] Massey H S W, McDaniel E W and Bederson B 1982 Applied Atomic Collision Physics vol 3 Gas Lasers (New York: Academic)

[2] Sze R C and Loree T R 1978 Experimental studies of a KrF and ArF discharge laser IEEE J. Quantum Electron. QE-14 944-50

[3] Levatter J I and Lin S C 1980 Necessary conditions for the homogeneous formation of pulsed avalanche discharges at high gas pressures J. Appl. Phys. 51 210-22

[4] Feenstra L, Hoekstra O B, Peters P J M and Witteman W J 2000 On the performance of an $\mathrm{ArF}$ and a $\mathrm{KrF}$ laser as a function of the preionisation timing and the excitation mode Appl. Phys. B 70 231-5

[5] Marchetti R, Penco E and Salvetti G 1984 A new type of corona-discharge photoionization source for gas lasers J. Appl. Phys. 56 3163-8

[6] Taylor R S and Leopold K E 1996 Surface corona discharge excited rare gas halide lamps Rev. Sci. Instrum. $672100-6$

[7] Edner H, Johansson J, Svanberg S and Wallinder E 1994 Fluorescence lidar multicolor imaging of vegetation Appl. Opt. 33 2471-9

[8] Fantoni R, Larciprete R, Piccirillo S, Bertoni G, Fratarcangeli R and Rotatori M 1988 Laser-induced dissociation of polychlorinated biphenyls in the liquid phase Chem. Phys. Lett. 143 245-50

[9] Esrom H and Kogelschatz U 1992 Modification of surfaces with new excimer UV sources Thin Solid Films 218 231-46

[10] Artana G and D'Adamo J 2002 Flow control with electrohydrodynamic actuators AIAA $J$. 40 1773-9

[11] Moreau E, Leger L and Touchard G 2006 Effect of a DC surface-corona discharge on a flat plate boundary layer for air flow velocity up to $25 \mathrm{~m} / \mathrm{s}$ J. Electrostat. $64215-25$

[12] Hidaka K and Murooka Y 1986 3.0-ns surface discharge development J. Appl. Phys. 59 87-92

[13] Hong S-K 2005 Electrical discharge plasma characteristics in pure Ar gas at multi-atmospheric pressure using the automatically pre-ionized plasma electrode $P h D$ Thesis Saga University, Saga, Japan

[14] Walter B 1985 Dielectrics for corona preionisation of a TEA laser J. Phys. E: Sci. Instrum. 18 279-81

[15] Kozlov M V, Sokolova M V, Temnikov A G, Timatkov V V and Vereshchagin I P 2003 Nanosecond surface corona discharge for different types of strip electrodes and ceramic or polymer dielectrics Plasmas Polym. 8 179-97

[16] Rutkevich I M 1986 Structure of a grazing discharge front Sov. Phys.-Tech. Phys 31 841-2

[17] Sobota A, van Dijk J, van Veldhuizen E M, Stoffels W W, Hendriks J, Manders F and M Haverlag 2007 Modelling of a pulsed argon discharge near a flat dielectric surface $28 \mathrm{th}$ ICPIG (Prague, Czech Republic, 15-20 July)

[18] Boeuf J P and Pitchford L C 2005 Electrohydrodynamic force and aerodynamic flow acceleration in surface dielectric barrier discharge J. Appl. Phys. 97103307

[19] Lay B, Moss R S, Rauf S and Kushner M J 2003 Breakdown processes in metal halide lamps Plasma Sources Sci. Technol. 12 8-21

[20] Scharfetter D L and Gummel H K 1969 Large-signal analysis of a silicon Read diode oscillator IEEE Trans. Electron Devices ED-16 64-77

[21] Kushner M J 2004 Modeling of microdischarge devices: pyramidal structures J. Appl. Phys. 95 846-59

[22] Li C, Ebert U, Brok W J M and Hundsdorfer W 2008 Spatial coupling of particle and fluid models for 
streamers: where nonlocality matters J. Phys. D: Appl. Phys. 41032005

[23] Li C, Ebert U and Hundsdorfer W 2009 3D hybrid computations for streamer discharges and production of run away electrons J. Phys. D: Appl. Phys. 42202003
[24] Rauf S and Kushner M J 1999 Dynamics of a coplanar-electrode plasma display panel cell: I. Basic operation J. Appl. Phys. 85 3460-9

[25] Lagarkov A N and Rutkevich I M 1994 Ionization Waves in Electrical Breakdown of Gases (New York: Springer) p 149 